\title{
Kiel 2019: geographischer Dialog fürr die Zukunft?
}

\author{
Matthew G. Hannah \\ Geographisches Institut, Universität Bayreuth, Bayreuth, Deutschland \\ Correspondence: Matthew G. Hannah (matthew.hannah@uni-bayreuth.de)
}

Received: 28 July 2020 - Revised: 26 August 2020 - Accepted: 26 August 2020 - Published: 9 October 2020

Kurzfassung. In conditions of unavoidably increasing mutual ignorance among human geographers about each others' areas of expertise, the question of how to pursue dialogue in a mutually respectful way that promotes insight and learning is ever more pressing. The dialogue between Carolin Schurr and Peter Weichhart in Kiel in 2019 offers instructive examples of how this issue can be addressed. The first, shorter part of the contribution is devoted to the substantive question of the unity of geographic inquiry, which was the subject matter of the dialogue. The bulk of the discussion, however, is devoted to analysing the dynamics of the dialogue itself, with a special focus on the sometimes very subtle and unintended ways in which positionalities leave their mark.

\section{Einleitung}

Wird es in 10 oder 20 Jahren rückblickend als wichtig erachtet werden, „dabei gewesen zu sein“ beim Dialog in Kiel im Oktober 2019 zwischen Carolin Schurr und Peter Weichhart? Bei was im Kontext des komfortablen akademischen Alltagsbetriebs, weit entfernt von den Entbehrungen und Gefahren der Feldforschung, wollen wir dabei sein? Genau bei solchen offenen Streitigkeiten, die laut dem „Mythos Kiel“ dort 1969 stattgefunden haben. Wir wollen mindestens ein bisschen aus der Komfortzone des Vorhersehbaren geworfen, überrascht, mit der Abbröckelung der üblichen Höflichkeiten konfrontiert werden.

Nach diesem Maßstab müsste Schurr vs. Weichhart Kiel 2019 enttäuschen: kein großer Streit; trotz wichtiger inhaltlicher Divergenzen zu viel Wohlwollen zwischen den Dialogpartner*innen. Aber gerade deswegen bin ich froh, dabei gewesen zu sein, weil die Art des Dialogs, der dort veranstaltet worden ist, zunehmend wichtig wird. Gerade deswegen verdient der Dialog selbst genau so viel Aufmerksamkeit wie die darin vertretenen Positionen.

Die wichtigste Frage, die sich aus dem Dialog zwischen Carolin Schurr und Peter Weichhart ergibt, lautet: „Wie sollte ein öffentlicher Dialog gestaltet werden, um die unvermeidlichen Wissensdefizite der unterschiedlichen Teilnehmenden möglichst respektvoll und einsichtsfördernd in Kontakt miteinander treten zu lassen?"Nach einem kurzen Ausflug in die inhaltliche Frage der disziplinären Kohärenz wird die Mehrheit des Beitrags dem Verlauf des Dialogs gewidmet.

\section{Kohärenz}

Schurr und Weichhart stellen einstimmig fest, dass die Vielfalt in der Geographie durch eine scheinbar unaufhaltsame Ausdifferenzierung nur noch größer wird. Damit wird es immer weniger möglich (wenn es überhaupt je möglich gewesen ist), dass einzelne Geographinnen und Geographen sich irgendeinen ausreichend informierten synthetischen Überblick über das gesamte Fach erarbeiten können. Teilweise als Ergebnis des Ausdifferenzierungsprozesses sei ein klares „Zentrum'“ in der Geographie nicht (mehr) auszumachen.

Beide im Kieler Dialog 2019 vorgetragenen Reaktionen auf diese Situation sind plausibel und vertretbar. Carolin Schurrs eher gelassene, ,zukunftsorientierte“ Würdigung der Vielfalt wird elegant mit ihrer Diskussion der Ideen from and of the margins exemplifiziert. Peter Weichharts eher ,herkunftsorientierter“ Vorschlag einer „Werkzeugskiste“, um eine Art „multiples Ersatz-Zentrum“"zusammenzuweben, steht in einer langen Tradition, deren Argumente nicht einfach von der Hand zu weisen sind (vgl. z. B. Jessop et al., 2008; zum Unterschied zwischen „Zukunfts-“ und „Herkunftsorientierung"vgl. Wardenga, 2020).

Ein einflussreicher Aufsatz von John Nystuen aus dem Jahr 1963 verdient in dieser Hinsicht unsere Aufmerksamkeit, weil er als Vergleichskulisse die Wichtigkeit des jewei- 
ligen Kontextes klarmacht. Mitten in der damaligen ,quantitativen Revolution“ in der anglophonen Geographie steckten Nystuens fachliche Kernbegriffe (direction oder orientation, distance, connection und relative position) eine $\mathrm{zu}-$ kunftsorientierte, abstrakte Perspektive ab, die gegen eine rückwärtsgewandte Überbetonung der Differenz und Einmaligkeit in der traditionellen Regionalgeographie ausgerichtet war (vgl. Nystuen, 1996 [1963]).

Die von Weichhart vorgeschlagenen Begriffe - die neben den drei schon von Nystuen ins Feld gebrachten Begriffen Distanz, Richtung und Konnektivität auch „Räumlichkeit (spatiality), Ort (place), Mobilität, Skalen, Kontextualität, Struktur, Prozess, Agency, Differenz, Disparitäten oder Grenze“ sowie wahlweise ,Agencements/Assemblages, Netzwerk, Rhizom oder das Konzept der sozialen Figurationen“ einschließen - sind ganz anders zeitlich gefärbt (vgl. Schurr and Weichhart, 2020:22). Sie stellen eine Art Rettungsversuch dar in einer Situation, wo ,unübersichtliche Differenz" eher mit Zukunft, dagegen Allgemeinheit und Zentralität mit Herkunft verbunden sind.

Carolin Schurr zeigt sich offen für Weichharts Grundidee, plädiert aber für eine flexible und im Prinzip potentiell grenzenlose Werkzeugkiste. Obwohl ich ihr voll zustimme, dass Exklusivität in Bezug auf Grundbegriffe gefährlich ist, würde ich selbst diese Gefahr laufen und Weichharts Liste als zu lang bewerten. Die Begriffe Kontextualität, Struktur, Prozess, Agency, Differenz, und Disparität spielen nämlich genauso wichtige Rollen in anderen Fächern wie der Geschichtswissenschaft oder der Soziologie - die Geographie kann keinen Anspruch auf diese Begriffe erheben. Gegenüber diesen Begriffen sollte sich die Geographie nicht verschließen. Sie sollten durchaus eine große Rolle spielen, aber als Transmissionsriemen oder Brücken zu benachbarten Disziplinen sowie zu disziplinübergreifenden Ansätzen auf der Ebene der Sozial- und Kulturtheorie.

Mein eigener Versuch, den lang (z. B. von Nystuen sowie von Weichhart) als zentral bezeichneten, aber trotzdem in der Forschung bisher fast vollständig vernachlässigten Grundbegriff „Richtung“ zu elaborieren und neu zu konzipieren, passt generell ins Programm von Weichhart (vgl. Hannah, 2019). Die Konzeption von Chris Philo, aufbauend zum Teil auf dem Ansatz der welfare geographies von David Smith aus den 1970er und 1980er Jahren sowie auf der Faschismuskritik der Frankfurter Schule, positioniert ,geographische Differenzierung" als zentralen Begriff (vgl. Philo, 2014, 2017; Chris Philo, persönliche Korrespondenz, 2020).

Philos Perspektive gründet auf der Einsicht, dass sich nicht nur der Faschismus im engeren Sinne, sondern die meisten Formen der Unterdrückung, Ausbeutung, Ausgrenzung und Ungerechtigkeit wesentlich aus Vereinfachungen (,Nation“, „Rasse“, „Volk“ usw.) speisen. Die Geographie kann nach Philo eine besonders ausgeprägte Expertise - und zwar über das ganze Spektrum von quantitativen und qualitativen Methoden - zur kritischen Analyse solcher Vereinfachungen in ihren räumlichen Ausprägungen vorweisen. Insofern stimmt
Philos Ansatz mit der von Schurr und Weichhart geteilten Meinung überein, die geographische Forschung solle sich nicht länger hinter der unhaltbaren Schimäre der ,Wertneutralität" verstecken, sondern zur Aufdeckung und Veränderung gesellschaftlicher Missstände ihren Beitrag leisten.

Diesen unterschiedlichen Kandidaten für Grundbegriffe der Geographie ist gemeinsam, dass sie alle Substantive sind. Es wäre auch wichtig zu fragen - nach dem alten Spruch „geography is what geographers do“-, ob es hilfreich wäre, das Problem des disziplinären Zusammenhalts mit Hilfe von Verben anzugehen. Diese Möglichkeit wird systematisch von Antje Schlottmann und Jeannine Wintzer ausgelotet. Ihr Buch „Weltbildwechsel: Ideengeschichten geographischen Denkens und Handelns" (vgl. Schlottmann und Wintzer, 2019) ist um Praktiken organisiert (Vermessen, Erklären, Erobern, Vermitteln, Aufklären, Wahrnehmen, Gestalten, Differenzieren, Visualisieren, Modellieren), die mit der Geographie lange Zeit verbunden gewesen sind.

Hier lohnt es sich m. E. auch zu fragen, wie viele von diesen Praktiken speziell ,geographisch“ sind; nicht um einige auszuschließen, sondern um ihre unterschiedlichen Rollen in der Disziplin klar zu machen. Vermessen, Erobern, Gestalten und Visualisieren wären dann primäre Kandidaten für einen disziplinären „Kern“. Die restlichen Praktiken wären dann nicht so sehr „Brückenbegriffe“ wie im Falle von Grundbegriffen in Form von Substantiven, sondern Anlässe klarzumachen, wie allgemeine wissenschaftliche Tätigkeiten speziell von Geographinnen und Geographen gehandhabt worden sind.

\section{Dialog, Positionalität und Unwissen}

Eine wichtige disziplinübergreifende wissenschaftliche Tätigkeit ist das Debattieren/Diskutieren. Einem Grundgedanken von Schlottmann und Wintzer folgend wäre es wichtig zu fragen, ob und inwiefern Debatten in der Geographie besondere Merkmale haben bzw. haben sollten. Für mich ist ein plausibler Kandidat für ein solches wünschenswertes Merkmal von Philo im übertragenen Sinne entlehnt: positionale Differenzierung. Eine Fähigkeit dazu ist ,geographisch“: Gerade in der Geographie sollte es uns leichtfallen, unterschiedliche diskursive und soziale Positionen differenziert zu „,kartieren“, statt sie pauschal unter einfachen master signifiers zusammenzupferchen (vgl. Rose, 1997). Das Spiel mit Visualisierungen des geographischen Diskurses als „Krapfen“ bzw. als „Donut“ könnte schon als Evidenz dafür verstanden werden.

Selbstverständlich ist Positionalität an sich weder einfach zu definieren noch allbestimmend. Wie Gillian Rose (1997) schon bemerkt hat, sind die quasi-kartographischen Vorstellungen hinter dem üblichen Gebrauch des Begriffs Positionalität zum Teil fragwürdig (vgl. Rose, 1997). Je näher betrachtet, desto schwieriger wird es, die Vielfalt positionaler Dimensionen (sozialer Status, geographische Lokation, 
ethnische, Geschlechts-, Alters-, institutionelle oder sonstige ,Standorte“) voneinander sauber zu entwirren oder sauber auf eine gemeinsame „Oberfläche“ zu projizieren, die eindeutige Vergleiche erlaubt.

Trotzdem lassen sich nach wie vor einige Dimensionen der Positionalität klar als Vermittler von relativen Vorteilen bzw. Nachteilen identifizieren. Aufgrund der eigenen Positionalität - in diesem Fall des eigenen Karrierewegs über drei Länder - fällt mir in der deutschsprachigen Geographie Geschlecht als eine solche besonders deutlich auf. Dabei geht es gerade nicht um die ,zählbaren“ Dinge wie die zunehmende Besetzung der Professuren und Lehrstühle durch Frauen, sondern um subtilere, i. d. R. unreflektierte Wertvorstellungen und Rollenerwartungen. Positionalität entfaltet ihre Wirkung, anders gesagt, nicht nur und oft nicht primär auf der Ebene der Absichten, Zielsetzungen, Personalpolitik usw.

Andererseits sind positionale Fragen nicht allbestimmend. In dieser Hinsicht bietet der veröffentlichte Dialog zwischen Reuben Rose-Redwood und Jonathan Smith einen erhellenden Vergleichspunkt zu denjenigem zwischen Schurr und Weichhart (vgl. Rose-Redwood and Smith, 2016). Obwohl die zwei Teilnehmer aus ähnlichen privilegierten Positionen argumentieren (weiße, männliche Inhaber sicherer akademischer Stellen) entwickelt sich ihr Dialog zu einer immer stärkeren Polarisierung zwischen inkompatiblen Perspektiven. Positionalität ist nicht Schicksal.

Was die Selektivität des positionierten Wissens streng impliziert - was aber in wissenschaftlichen Kreisen eher ungern in den Vordergrund gestellt wird -, ist, dass jede Position automatisch und untilgbar mit spezifischen Formen des Unwissens verbunden ist. Unser Unwissen als solches kann nicht „überwunden“ werden, höchstens dehnen wir unser Wissen mühsam durch Lektüre, empirische Forschung und anderweitige wissenschaftliche Tätigkeiten bescheiden aus. Das oft beschworene kumulative Wachstum des allgemeinen Wissensbestandes ist kein Trost, ganz im Gegenteil: Wir können selbst nur einen winzigen - und mit dem sich beschleunigenden Wachstum dieses Wissensbestandes immer kleiner werdenden - Bruchteil davon selbst detailliert in Erfahrung bringen.

Die Aufteilung der Wissenschaft in einzelne Disziplinen ist hier zunächst eine praktische Hilfe. Die Grenzen einer Disziplin machen gleichzeitig die äußerste Grenze dessen aus, wofür wir im Bereich der Wissensaneignung einigermaßen ,verantwortlich“ sind oder gemacht werden können. Wenn es aber, wie in der Geographie, für die einzelnen Wissenschaftler*innen nicht mehr möglich ist, sich einen gleichzeitig flächendeckenden und ausreichend tiefgehenden Überblick über alle Forschungsrichtungen zu verschaffen, müssen alle Dialoge und Auseinandersetzungen zwischen unterschiedlich positionierten Wissenschaftler*innen über die Kohärenz oder Richtung der Gesamtdisziplin zu einem erheblichen Grad aus gegenseitiger Ignoranz geführt werden. Dies ist der engere Ausgangspunkt für den hiesigen Beitrag. Die bloße Präsenz der Wissensdefizite seitens aller Teilneh- mer*innen ist an sich eher uninteressant, weil absolut unvermeidlich. Die Frage ist: Wie gehen sie damit um?

Hier können wir vom Dialog zwischen Carolin Schurr und Peter Weichhart durchaus etwas lernen. Wie anfangs erwähnt, erhoffen sich viele Zuschauer*innen in einer solchen Situation wie in Kiel 2019 „klare Kanten“, etwas Krach. Wenn wir aber in Erwägung ziehen, dass die unterschiedlichen Positionalitäten und Wissensbestände der Dialogpartner*innen zwangsläufig auch große Wissensdefizite gegenüber der jeweils anderen Position implizieren, ist die Gefahr groß - ich meine: sehr groß! -, dass Krach und klare Gegenüberstellungen weitgehend auf nicht ausreichend begründeten Meinungen fußen.

Je nach akademischer Ausbildung und Erfahrungen - je nach Position - kennen wir alle pauschale Verurteilungen von Ansätzen, bei denen wir selbst wissen, dass die urteilenden Kolleg*innen entweder selbst wenig bis nichts über den betroffenen Ansatz gelesen bzw. erforscht haben, oder wenn doch (um ganz großzügig gegenüber diesen Kolleg*innen zu sein), dass sie es offensichtlich mit solchen eisernen Scheuklappen getan haben, dass sie den Ansatz am Ende viel zu unterkomplex verstanden haben. Um es etwas überspitzt zu formulieren, das Muster der drastischen Vereinfachung der Differenzen, das Chris Philo als ideologische Wurzel vielen Unheils im politischen Bereich identifiziert, ist in der wissenschaftlichen Debatte analog schädlich.

Zum großen Glück ist der Dialog zwischen Carolin Schurr und Peter Weichhart nicht in eine solche wissenschaftlich unverantwortliche Richtung ab- bzw. auseinandergedriftet. Stattdessen haben beide zwei Tugenden zutage gebracht, die aus einem Sinn für positionale Differenzierung erwachsen: Bescheidenheit, was das Ausmaß und den Geltungsbereich des eigenen Wissens angeht, und genuine Neugier gegenüber dem bisher Unbekannten.

In den letzten Jahren ist - oft in Anlehnung an die Ideen von Chantal Mouffe - immer wieder die Legitimität des „Agonismus“ im Rahmen von Dialog betont worden (vgl. Rose-Redwood et al., 2018). Strukturell gesehen aber läuft die Haltung des Agonismus in tendenziell wachsender Ermangelung von ausreichendem Wissen über die jeweils andere Position Gefahr, Lerneffekte eher auszuschließen als zu erzeugen.

Carolin Schurr drückt die oben erwähnten Tugenden in ihren Fragen an Peter Weichhart aus, wie es war, zur Zeit des Kieler Kongresses 1969 als Geograph unterwegs gewesen zu sein, sowie in ihrer Offenheit für seinen WerkzeugkistenVorschlag. Peter Weichhart zeigt sich für Carolin Schurrs Argumentation offen, dass gerade Perspektiven am Rande der Disziplin (und der disziplinären Macht) besonders fruchtbar sein können. Die Übereinstimmung darüber, dass Fragen der (Un)gerechtigkeit eine Art forschungsrichtungsübergreifendes Bindeglied in der Humangeographie bilden könnten, setzt eine fundamental konstruktive gegenseitige Offenheit voraus. 


\section{Schattierungen der Offenheit}

Wenn wir - wie ich meine - zunehmend Dialoge und Debatten aufgrund von Ignoranz oder Unwissen führen müssen, reicht es aber nicht, Carolin Schurr und Peter Weichhart für ihre vorbildliche gegenseitige Offenheit zu gratulieren. Diese Offenheit sollte zwar nicht unterschätzt werden: Wie oben schon argumentiert, ist sie viel besser als eine allzu häufig praktizierte (um es höflich auszudrücken) ,unterinformierte" Kampfhaltung. Es lohnt sich trotzdem, uns etwas genauer und detaillierter die unterschiedliche Art und Weise anzuschauen, in der diese Tugenden von den beiden realisiert worden sind. Dabei treten interessante Asymmetrien zu Tage.

Mich interessiert im Folgenden vor allem die Art und Weise, in der Peter Weichhart seinen Part im Dialog gespielt hat. Hier geht es mir nicht um eine persönliche Kritik: Ich bewundere seine Arbeit und schätze seine Art der Interaktion mit Kolleg*innen jenseits dieses Dialogs. Die Positionalität von Peter Weichhart ist auch nicht leicht auf einen Nenner zu bringen. Bei ihm wie bei uns allen kreuzen sich unterschiedliche Dimensionen auf komplizierte Art und Weise (siehe oben). Er versteht sich selbst im Rahmen des Dialogs offensichtlich nicht als positional ,privilegiert“. Trotzdem schreibe ich ihm (wie übrigens mir selbst) wesentliche positionale Privilegien $\mathrm{zu}$, die sich trotz seiner Intention auf unreflektierter, subtiler Ebene ausdrücken. Gerade weil seine privilegierte Position nicht gewollt und nicht offensichtlich ist - wie bei vielen von „uns“ Lupe zu nehmen.

Der Hintergrund für diese Fokussierung lässt sich grob mit dem Hinweis auf eine sehr wichtige, grundlegende Einsicht vieler „Rand-“ bzw. „subalternen“ Theorietraditionen andeuten, die zum Beispiel in der feministischen Theorie als „Standpunktepistemologie“ (standpoint epistemology) bezeichnet wird (vgl. McDowell, 1992). Ob mit primärem Bezug auf Geschlecht, Ethnizität, Behinderung oder intersektionale, hierarchisierende Konstrukte anderer Art - die Grundidee der Standpunktepistemologie ist, dass Mitglieder subalterngemachter oder ausgeschlossener Gruppen generell bessere Möglichkeiten als die Herrschenden haben, die Machtsysteme, durch die sie marginalisiert oder diskriminiert werden, zu durchblicken. Dies ist nicht zuletzt deswegen der Fall, weil Mitglieder der dominanten Gruppen i. d. R. kein oder weniger Interesse daran haben, die ungerechte Basis oder die oft subtilen Mechanismen hinter den eigenen Privilegien oder Vorteilen klar zu erkennen.

Grob gesagt, Kolleg*innen wie Carolin Schurr, die aus einfachen oder intersektional verdichteten ,subalternen“ Positionen ihren Weg durch die Wissenschaft machen müssen, haben i. d. R. eine klarere Sicht auf die disziplinären Machtmechanismen, nicht zuletzt auf die gestaltende Wirkung von Positionalität jenseits der universell anerkannten Wichtigkeit der hierarchischen Beziehungen zwischen Professor*innen und niedrigeren Statusgruppen.
So fällt es Carolin Schurr strukturell gesehen leichter, sich zum Beispiel das Muster zu merken, wonach es im Grunde ausschließlich weiße Männer sind, die die Unmöglichkeit eines allumfassenden synthetischen Blicks über das Fach als problematisch empfinden (vgl., Schurr and Weichhart, 2020:24). Peter Weichhart ist natürlich auch fähig, dieses Muster zu erkennen, aber anders als bei Carolin Schurr verlangt es von ihm einen extra, unforcierten Schritt der ,SelbstProvinzialisierung“, worin viele Mitglieder der dominanten Gruppen immer noch relativ wenig Übung haben.

Wichtig ist hier m. E. der Umgang mit dem eigenen Nichtwissen. Ganz im Sinne des seit Jahrzehnten kritisierten erhöhten Leistungsdrucks spüren Mitglieder der unterrepräsentierten Gruppen in der Wissenschaft einen stärkeren Druck, die unvermeidlichen Lücken im eigenen Wissensbestand doch möglichst weitgehend zu schließen. Bei Mitgliedern der dominanten Kategorien ist dagegen der schnellstmögliche und fortdauernde Ausbau des eigenen Wissensvorrats und der entsprechende Abbau der Ignoranz nicht unbedingt so dringend.

Daraus ergibt sich m. E. eine subtile Differenz in der typischen Haltung zum eigenen Unwissen, die im Austausch zwischen Schurr und Weichhart auch zu sehen ist. Die Neugier, die Carolin Schurr gegenüber Peter Weichharts Erfahrungen rund um die Kieler Ereignisse 1969 zeigt, ist zweifellos genuin, nicht aber unbedingt optional. Hätte sie sich gegenüber den damaligen Vorgängen und Erfahrungen indifferent oder demonstrativ uninformiert gezeigt, wäre das wahrscheinlich von großen Teilen des Publikums als unverantwortlich verstanden worden. Das heißt, sie fühlte sich vermutlich zu einem gewissen Grad dazu verpflichtet, sich im Vorfeld so gut wie möglich über Kiel 1969 zu informieren - sofern sie es nicht schon vorher gewesen ist - und als gut vorbereitet aufzutreten.

Bei Peter Weichhart kann man zwar nicht eine gegenteilige Haltung feststellen. Er trat im Kieler Dialog überhaupt nicht überheblich auf, gab unter anderem zu, dass er selbst 1969 in Kiel nicht dabei gewesen ist, zeigte auch für viele Ideen und Perspektiven, die Carolin Schurr ins Feld geführt hat, genuine Offenheit und Neugier. Er hat auch durch die Erwähnung von Begriffen wie „Assemblage“ gezeigt, dass er neuere Ansätze nicht einfach ignoriert. Trotzdem sind seine Beiträge zum Dialog deutlich weniger geprägt von einem positionellen Drang, eine solide Wissensbasis in den für seine eigene Forschung eher weniger relevanten Bereichen auf den Tisch zu legen.

Dies ist am klarsten in seiner Diskussion der unüberschaubar gewordenen Vielfalt der Ansätze sichtbar. Seinen vielgelesenen, 2008 veröffentlichten Überblick über die Sozialgeographie zitierend, meint er, dass wir uns als Geographinnen und Geographen ,auf einer großen Spielwiese“ befinden ,auf der sich zahlreiche wunderschöne Steckenpferdchen tummeln, deren Bewegungen aber weitgehend unkoordiniert und ohne gemeinsame Choreographie erfolgen. Jedes dieser prächtigen Steckenpferde ist an spannenden Ein- 
zelthemen und zweifellos sehr wichtigen Fragen orientiert" (vgl. Schurr and Weichhart, 2020:9, zit. nach Weichhart, 1998:410).

Die unterschwellige Gelassenheit gegenüber den Grenzen des eigenen Wissens ist hier evident. Nicht nur der ästhetisierende Außenblick (,wunderschöne“, ,prächtigen“), sondern auch die Phrasen ,spannenden Einzelthemen“ und ,,zweifellos sehr wichtigen Fragen" beweisen eine Haltung, wonach die eigenen Wissenslücken für die Legitimität der eigenen Perspektive nicht abträglich sind, besonders, wenn man die einem selbst nicht im Detail vertrauten Ansätze trotz mangelnden Wissens wohlwollend-positiv bewundert.

Diese Haltung an sich macht das Problem nicht aus. Wie oben ausgeführt, ist ein wachsendes Unwissen gegenüber anderen Ansätzen unvermeidlich. Da ist es viel besser, die nicht vertrauten Perspektiven irgendwie positiv und offen zu betrachten, als abschätzig ohne vertretbaren Grund abzuschmettern. Aber diese Haltung ist erstens positional bestimmt: Carolin Schurr hätte sich so eine Haltung nicht leisten können.

Zweitens, und ganz zentral für mein Argument, können wir uns in solchen Dialogsituationen nicht allein auf wohlwollende Absichten verlassen. Obwohl bestimmt gar nicht so gemeint, trivialisieren Weichharts Formulierungen die Ansätze, die er als Nicht-Experte von außen betrachtet. Um diese Trivialisierung festzustellen, bedarf es keiner besonders raffinierten Dekonstruktionsarbeit, ein Blick in den Duden reicht. Der Duden liefert als zweite, figurative Definition von „Steckenpferd“ Folgendes: „von Außenstehenden leicht als [liebenswürdige] Schrulle belächelte Liebhaberei, der jemand seine freie Zeit widmet" (vgl. Duden, 2020). Das von Weichhart benutzte Suffix ,-chen“ (,Steckenpferdchen“) verstärkt die Trivialisierung zusätzlich.

Diese Bedeutung unterstützt die ganzen, weniger gut gemeinten Pseudokritiken, wonach wahlweise der Poststrukturalismus, der Postkolonialismus, queere Geographien oder andere Ansätze irgendwie nicht ernsthafte wissenschaftliche Forschungsrichtungen seien, sondern Varianten einer perversen, narzisstischen Art von nutzloser Wortspielerei. Weichharts respektvolle persönliche Teilnahme am Dialog wird hier ziemlich klar und gegen seinen Willen durch die respektlosen Formulierungen untergraben.

Gerade anhand seines in meiner Erfahrung ungewöhnlich offenen und konstruktiven Auftritts ist dies zutiefst bedenklich. Der Dialog zeugt von der dringenden Notwendigkeit, dass wir uns im Kontext des zunehmenden gegenseitigen Nichtwissens bzgl. der Details anderer Ansätze nicht mit der Feststellung zufriedengeben können, dass wir es ,gut meinen", oder dass wir wohlwollend mit anderen in Dialog treten. Wir müssen einen Schritt weitergehen und unseren Sprachgebrauch ständig und reflexiv überprüfen. Sonst laufen wir Gefahr, unbewusste, ungewollte und uns selbst sowie den Adressat*innen im Grunde unwürdige Unterstellungen über Kolleg*innen durch die Hintertür einzulassen.
Im Kieler Dialog 2019 sehen wir also m. E. einerseits einen Austausch, in dem die von beiden Teilnehmer*innen praktizierte Offenheit und Neugier in einer Situation der gegenseitigen Teil-Ignoranz Grund für „zukunftsorientierte“ Hoffnung geben (Wardenga, 2020). Andererseits ist es im Dialog auch klar, dass gute, menschliche Absichten - besonders seitens Mitgliedern der dominanten Gruppe - nicht ausreichen. Der reflektierte Sinn für positionale Differenzierung, der gerade geographische Debatten besonders respektvoll und konstruktiv machen sollte, muss mit intensiverer sprachlicher Reflexion unterstützt werden.

Datenverfüg barkeit. Für diesen Artikel wurden keine Datensätze genutzt.

Interessenkonflikt. Der Autor erklärt, dass kein Interessenkonflikt besteht.

Danksagung. Der Autor möchte Nadine Marquardt und Benedikt Korf für die Einladung zur Einreichung dieses Beitrags danken und außerdem dankt er Nadine Marquardt und einem/einer anonymen Gutachter*in für ihre hilfreichen Kommentare und Kritik.

Begutachtung. This paper was edited by Nadine Marquardt and reviewed by one anonymous referee.

\section{Literatur}

Duden: Steckenpferd, online aufrufbar: https://www.duden.de/ rechtschreibung/Steckenpferd, letzter Zugriff: 23. Juli 2020.

Hannah, M.: Direction and Socio-Spatial Theory: A Political Economy of Oriented Practice, Routledge, London, 2019.

Jessop, B., Brenner, N., and Jones, M.: Theorizing sociospatial relations, Environ. Plan. D, 26, 389-401, 2008.

McDowell, L.: Doing Gender: Feminism, Feminists and Research Methods in Human Geography, Trans. Inst. Brit. Geogr., 17, 399-416, 1992.

Nystuen, J.: Identification of some fundamental spatial concepts, in: Human Geography: An Anthology, edited by: Agnew, J., Livingstone, D., and Rodgers, A., Blackwell, Oxford, 590-599, 1996 [1963].

Philo, C.: Well-being, mental health and the Smiths, in: Annual Lecture, edited by: Smith, D. M., Queen Mary University of London, School of Geography, London, 26 November 2014.

Philo, C.: Less-than-human geographies (Guest Editorial), Polit. Geogr., 60, 256-258, 2017.

Rose, G.: Situated knowledges: positionality, reflexivities and other tactics, Prog. Human Geogr., 21, 305-320, 1997.

Rose-Redwood, R. and Smith, J.: Strange encounters: a dialogue on cultural geography across the political divide, J. Cult. Geogr., 33, 356-378, 2016. 
Rose-Redwood, R., Kitchen, R., Rickards, L., Rossi, U., Datta, A., and Crampton, J.: The possibilities and limits to dialogue, Dialog. Human Geogr., 8, 109-123, 2018.

Schlottmann, A. und Wintzer, J.: Weltbildwechsel: Ideengeschichten geographischen Denkens und Handelns, UTB, Bern, 2019.

Schurr, C. and Weichhart, P.: From Margin to Center? Theoretische Aufbrüche in der Geographie seit Kiel 1969, Geogr. Helv., 75, 53-67, https://doi.org/10.5194/gh-75-53-2020, 2020.
Wardenga, U.: Vergangene Zukünfte - oder: Die Verhalndlung neuer Möglichkeitsräume in der Geographie, Geogr. Z., 108, 4-22, 2020.

Weichhart, P.: Entwicklungslinien der Sozialgeographie: von Hans Bobek bis Benno Werlen, Franz Steiner Verlag, Stuttgart, 1998. 